\title{
MHD Thin Film Flows of a Third Grade Fluid on a Vertical Belt with Slip Boundary Conditions
}

\author{
Taza Gul, ${ }^{1}$ Rehan Ali Shah, ${ }^{2}$ Saeed Islam, ${ }^{1}$ and Muhammad Arif ${ }^{1}$ \\ ${ }^{1}$ Department of Mathematics, Abdul Wali Khan University Mardan, Mardan, Pakistan \\ ${ }^{2}$ Department of Mathematics, UET, Peshawar, Pakistan \\ Correspondence should be addressed to Taza Gul; tazagulsafi@yahoo.com
}

Received 23 May 2013; Accepted 14 August 2013

Academic Editor: Oluwole Daniel Makinde

Copyright (C) 2013 Taza Gul et al. This is an open access article distributed under the Creative Commons Attribution License, which permits unrestricted use, distribution, and reproduction in any medium, provided the original work is properly cited.

\begin{abstract}
The problem of heat transfer analysis is considered in electrically conducting thin film flows with slip boundary conditions. The flow is assumed to be obeying the nonlinear rheological constitutive equation of a third grade fluid. We have solved the governing nonlinear equations of present problems using the traditional Adomian decomposition method (ADM). Particular attention is given to the combined effect of heat and MHD on the velocity field. The results include the profile of velocity, volume flux, skin friction, average velocity, and the temperature distribution across the film. The effects of model parameters on velocity, skin friction and temperature variation have been studied. Optimal homotopy asymptotic method (OHAM) is also used for comparison. The numerical results and absolute errors are derived in tables.
\end{abstract}

\section{Introduction}

The flow and heat transfer inside thin films are ubiquitous in civil, environmental sciences, mechanical engineering, biological sciences, geophysics, and elsewhere. This is due to their several applications at large scale such as wire and fiber coating, reactor fluidization, paper production, different food stuffs like ketchup, sauce, and honey, transpiration cooling, gaseous diffusion, drilling mud, oil wills, heat pipes, and fluid cells. The problem of chambers for chemical and biological detection systems like fluid of many chemicals was considered by Lavrik et al. [1]. Heat transfer inside thin film flow with variable pressure was discussed by Khaled and Vafai [2]. Thin film unsteady flow with variable viscosity was investigated by Nadeem and Awais [3]. Munson and Young [4] discussed the thin film flow of Newtonian fluids. Alam et al. investigated the thin film flow of Johnson-Segalman fluids for lifting and drainage problems [5]. The MHD thin film flow was discussed by Hameed and Ellahi for a non-Newtonian fluid on a vertical moving belt [6]. Due to complexity of non-Newtonian fluids, it becomes difficult to suggest a single model which exhibits all properties of non-Newtonian fluids; therefore various empirical and semiempirical models have been imposed. The use of heat transfer together with the
MHD and non-Newtonian fluids under the influence of slip boundary conditions is of particular interest in chemical processing. Relevant and interesting work about present work may be found in $[7,8]$. One of the established models amongst non-Newtonian fluids is class of third grade fluids which have their constitutive equations based on strong theoretical foundations, where the relation between the stress and strain is not linear. To solve real world problems, different approximate techniques have been used in mathematics, fluid mechanics, and engineering sciences [9]. Some of the common methods are VIM [10-14], DTM [15, 16], HPM [17-19], HAM, and OHAM [20-24]. These methods deal with the nonlinear problems effectively. The work under various configurations on the thin film flows was discussed by Siddiqui et al. [25]. In [26] they examined the thin film flows of Sisko and Oldroyd6 constant fluids on a moving belt. The heat transfer analysis of thin film is also discussed by Chakraborty and Som [27]. The main aim of the present work is to study heat transfer into a thin film of a third grade fluid on a vertical belt under the influence of transverse magnetic field with slip boundary conditions using ADM. In 1992 Adomian [28, 29] introduced the ADM for the approximate solutions for linear and nonlinear problems. Wazwaz [30, 31] used ADM for the reliable treatment of Bratu-type and Emden-Fowler equations. 


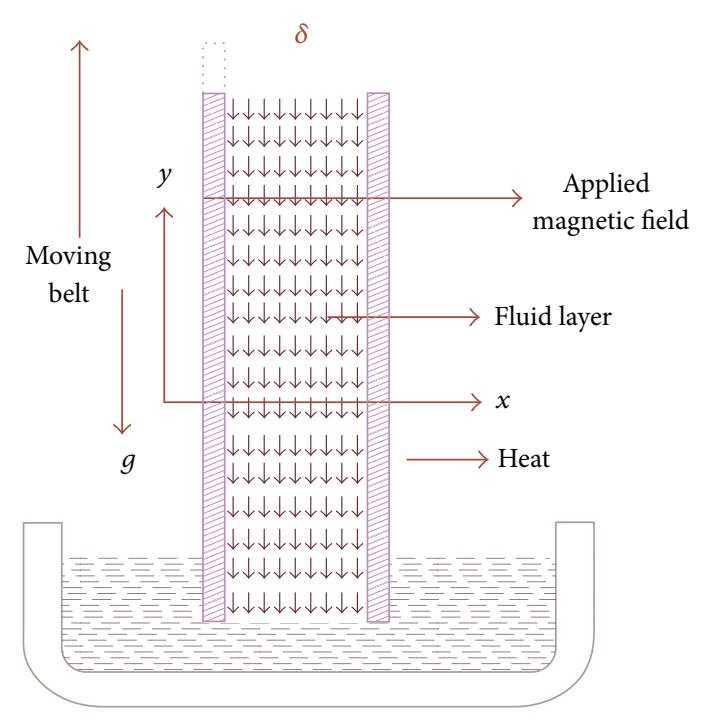

FIGURE 1: Geometry of the lifting problem.

\section{Basic Equations}

The governing equations of an incompressible isothermal and electrically conducting third grade fluid are

$$
\begin{gathered}
\nabla \cdot \mathbf{U}=\mathbf{0}, \\
\rho \frac{D \mathbf{U}}{D t}=\nabla \cdot \mathbf{T}+\rho \mathbf{g}+\mathbf{J} \times \mathbf{B}, \\
\rho c_{p} \frac{D \boldsymbol{\Theta}}{D t}=\kappa \nabla^{2} \boldsymbol{\Theta}+\operatorname{tr}(\boldsymbol{\tau} \cdot \mathbf{L}),
\end{gathered}
$$

where $\rho$ is the constant density, $\mathbf{g}$ is body force per unit mass, $\mathbf{U}$ is velocity vector of the fluid, $\boldsymbol{\Theta}$ is temperature, $\kappa$ is thermal conductivity, $c_{p}$ is specific heat, $\mathbf{L}=\nabla \mathbf{U}, D / D t=\partial / \partial t+(\mathbf{U} \cdot \nabla)$ denote material time derivative, $\boldsymbol{\tau}$ is Cauchy stress tensor, and $\mathbf{T}$ is the shear stress. A uniform magnetic field $\mathbf{B}=\left(0, B_{0}, 0\right)$ is imposed transversely on the belt. The Lorentz force per unit volume is given by

$$
\mathbf{J} \times \mathbf{B}=\left[0, \sigma B_{0}^{2} u(x), 0\right] .
$$

Shear stress tensor $\mathbf{T}$ is given by

$$
\mathbf{T}=-p \mathbf{I}+\boldsymbol{\tau}
$$

where $-p \mathbf{I}$ denotes spherical stress and $\boldsymbol{\tau}$ is defined as

$$
\begin{aligned}
\boldsymbol{\tau}= & \mu \mathbf{A}_{1}+\alpha_{1} \mathbf{A}_{2}+\alpha_{2} \mathbf{A}_{1}^{2}+\beta_{1} \mathbf{A}_{3} \\
& +\beta_{2}\left(\mathbf{A}_{1} \mathbf{A}_{2}+\mathbf{A}_{2} \mathbf{A}_{1}\right)+\beta_{3}\left(\operatorname{tr} \mathbf{A}_{1}^{2}\right) \mathbf{A}_{1} .
\end{aligned}
$$

Here $\alpha_{i}$ and $\beta_{j}$ are the material constants, and $\mathbf{A}_{1}, \mathbf{A}_{2}$, and $\mathbf{A}_{3}$ are the kinematical tensors given by

$$
\begin{array}{r}
\mathbf{A}_{n}=\frac{D \mathbf{A}_{n-1}}{D t}+\mathbf{A}_{n-1}(\nabla \mathbf{u})+(\nabla \mathbf{u})^{T} \mathbf{A}_{n-1}, \quad n \geq 1, \\
\mu \geq 0, \quad \alpha_{1} \geq 0, \quad\left|\alpha_{1}+\alpha_{2}\right| \leq \sqrt{24 \mu \beta_{3}}, \quad \beta_{3} \geq 0 .
\end{array}
$$

\section{Formulation of the Lift Problem}

Consider a wide flat belt moving vertically upward at constant speed $U_{0}$, through a large bath of third grade liquid as shown in Figure 1. The belt carries with it a layer of liquid of constant thickness, $\delta$. For analysis coordinate system is chosen, in which the $x$-axis is taken parallel to the surface of the belt and $y$-axis is perpendicular to the belt. Uniform magnetic field is applied transversely to the belt. Assume the flow is steady and laminar after a small distance above the liquid surface layer. The external pressure is atmospheric everywhere.

Velocity and temperature fields are

$$
\mathbf{u}=(0, u(x), 0), \quad \Theta=\Theta(x) .
$$

Boundary conditions are

$$
\begin{gathered}
u=U_{0}-\gamma T_{x y} \quad \text { at } x=0, \\
\frac{d u}{d x}=0, \quad \text { at } x=\delta, \\
\Theta=\Theta_{0}, \quad \text { at } x=0, \\
\Theta=\Theta_{1}, \quad \text { at } x=\delta .
\end{gathered}
$$

Inserting the velocity field given in (9) in (1) and (5)-(7), the continuity equation (1) satisfies identically and (5) gives the following components of stress tensor

$$
\begin{gathered}
T_{x x}=-P+\left(2 \alpha_{1}+\alpha_{2}\right)\left(\frac{d u}{d x}\right)^{2}, \\
T_{x y}=\mu \frac{d u}{d x}+2\left(\beta_{2}+\beta_{3}\right)\left(\frac{d u}{d x}\right)^{3}, \\
T_{y y}=-P+\alpha_{2}\left(\frac{d u}{d x}\right), \\
T_{z z}=-P, \\
T_{x z}=T_{y z}=0 .
\end{gathered}
$$
to

Using (11), the momentum and energy equations reduce

$$
\begin{gathered}
0=\mu \frac{d^{2} u}{d x^{2}}+6\left(\beta_{2}+\beta_{3}\right)\left(\frac{d u}{d x}\right)^{2}\left(\frac{d^{2} u}{d x^{2}}\right)-\rho g-\sigma B_{0}^{2} u(x), \\
0=\kappa \frac{d^{2} \Theta}{d x^{2}}+\mu\left(\frac{d u}{d x}\right)^{2}+2\left(\beta_{2}+\beta_{3}\right)\left(\frac{d u}{d x}\right)^{4}
\end{gathered}
$$


Introducing the following nondimensional variables:

$$
\begin{aligned}
& \bar{u}=\frac{\delta}{\nu} U, \quad \bar{x}=\frac{x}{\delta}, \quad \bar{\Theta}=\frac{\Theta-\Theta_{0}}{\Theta_{1}-\Theta_{0}}, \\
& \bar{\gamma}=\frac{\mu \gamma}{\delta}, \quad \Lambda=\frac{\gamma \nu}{\delta}, \\
& \lambda=\frac{\mu \nu^{2}}{k\left(\Theta_{1}-\Theta_{0}\right) \delta^{2}}, \quad m=\frac{\delta^{3} g}{\nu^{2}}, \\
& M=\frac{\sigma B_{0}^{2} \delta^{2}}{\mu}, \quad \beta=\frac{\left(\beta_{2}+\beta_{3}\right) \nu^{2}}{\mu \delta^{4}}, \\
& \alpha=\frac{\delta U_{0}}{\nu}, \quad R_{e}=\frac{U \delta}{\nu}, \quad \nu=\frac{\mu}{\rho},
\end{aligned}
$$

where $m$ is the gravitational parameter, $M$ is magnetic parameter, $\beta$ is non-Newtonian effect, $\Lambda$ is slip parameter, $\lambda$ is heat dimensionless number, $R_{e}$ is the local Reynolds number, and $\alpha$ is the nondimensional variable using the above dimensionless variables in (10) and in (12) and dropping bars we obtain

$$
\begin{gathered}
\frac{d^{2} u}{d x^{2}}+6 \beta\left(\frac{d u}{d x}\right)^{2}\left(\frac{d^{2} u}{d x^{2}}\right)-m-M u(x)=0 \\
\frac{d^{2} \Theta}{d x^{2}}+\lambda\left[\left(\frac{d u}{d x}\right)^{2}+2 \beta\left(\frac{d u}{d x}\right)^{4}\right]=0 \\
u_{n}(0)=\alpha-\Lambda\left(\frac{d u_{n}}{d x}+2 \beta\left(\frac{d u_{n}}{d x}\right)^{3}\right) \\
\frac{d u_{n}(1)}{d x}=0, \quad n=0 \\
u_{n}(0)=-\Lambda\left(\frac{d u_{n}}{d x}+2 \beta\left(\frac{d u_{n}}{d x}\right)^{3}\right) \\
\frac{d u_{n}(1)}{d x}=0, \quad n>0 \\
\Theta(0)=0, \quad(1)=1 .
\end{gathered}
$$

\section{Adomian Decomposition Method (ADM)}

A general description of the method is as follows. Begin with an equation $\widetilde{F}(u)=\widetilde{g}(x)$, where $\widetilde{F}$ represents a general nonlinear ordinary differential operator involving both linear and nonlinear terms and $\widetilde{g}$ is a source term. The linear term is decomposed into $\widetilde{L}+\widetilde{R}$, where $\widetilde{L}$ is easily invertible and $\widetilde{R}$ is the remainder of the linear operator. For convenience, $\widetilde{L}$ may be taken as the highest order derivative which avoids difficult integrations which result when complicated Green's functions are involved. Thus the equation may be written as follows:

$$
\widetilde{L} u+\widetilde{R} u+\widetilde{N} u=\widetilde{g} .
$$

Solving for $\widetilde{L} u$,

$$
\widetilde{L} u=\widetilde{g}-\widetilde{R} u-\widetilde{N} u,
$$

where $\widetilde{N}$ is non-linear operator and $\widetilde{g}$ is a source term, since $\widetilde{L}$ is easily invertible.

Equation (20) can be written as follows:

$$
\widetilde{L}^{-1} \widetilde{L} u=\widetilde{L}^{-1} \widetilde{g}-\widetilde{L}^{-1} \widetilde{R} u-\widetilde{L}^{-1} \widetilde{N} u .
$$

We use $\widetilde{L}^{-1}$ depending on the order of the differential equation. If differential equation is an initial-value problem, if it is desired for boundary value problem as well, integrations are used. The constants of integration are evaluated from the given initial and boundary conditions. $\widetilde{L}^{-1}$ can also be treated as definite integral from $\left(\widetilde{t}_{0}\right.$ to $\left.\widetilde{t}\right)$. Solving (21) for $u$ we obtained

$$
u=C_{1}+C_{2} t+\widetilde{L}^{-1} \tilde{g}-\widetilde{L}^{-1} \widetilde{R} u-\widetilde{L}^{-1} \widetilde{N} u
$$

The non-linear term $\widetilde{N} u$, is defined as follows:

$$
\widetilde{N} u=\sum_{k=0}^{\infty} \widetilde{A}_{n}
$$

Here $\widetilde{A}_{n}$ are special polynomials called Adomian polynomials and $\widetilde{u}$ will be equated to $\sum_{k=0}^{\infty} \widetilde{u}_{n}$. The initial velocity is identified as follows:

$$
\begin{gathered}
\widetilde{u}_{0}=\widetilde{C}_{1}+\widetilde{C}_{2} t+\widetilde{L}^{-1} \widetilde{g} \\
\sum_{k=0}^{\infty} u_{n}=u_{0}-\widetilde{L}^{-1} \widetilde{R} \sum_{k=0}^{\infty} u_{n}-\widetilde{L}^{-1} \sum_{k=0}^{\infty} \widetilde{A}_{n} .
\end{gathered}
$$

Comparison for different components of velocity profile is as follows:

$$
\begin{gathered}
u_{1}=-\widetilde{L}^{-1} \widetilde{R} u_{0}-\widetilde{L}^{-1} \widetilde{A}_{0}, \\
u_{2}=-\widetilde{L}^{-1} \widetilde{R} u_{0}-\widetilde{L}^{-1} \widetilde{A}_{1}, \\
\vdots \\
u_{n+1}=-\widetilde{L}^{-1} \widetilde{R} u_{n}-\widetilde{L}^{-1} \widetilde{A}_{n} .
\end{gathered}
$$

The Adomian polynomials $\widetilde{A}_{n}$ depend on the velocity components $u_{0}, u_{1}, u_{2}, \ldots, u_{n}$, which play a flourishing role in the rapid convergence of the series. In the above series $\widetilde{A}_{0}$ depends only on $u_{0}, \widetilde{A}_{1}$ depends on $u_{0}$ and $u_{1}, \widetilde{A}_{2}$ depends on $u_{0}, u_{1}$, and $u_{2}$, and so forth. Relevant discussion about ADM can be seen in $[16,17]$.

4.1. The ADM Solution of Lifting Problem. Using the inverse operator $\widetilde{L}^{-1}=\iint d u^{\prime}$ of the Adomian decomposition method on (14), we obtained

$$
u=C_{1} x+C_{2}+m \frac{x^{2}}{2}+\widetilde{L}^{-1}[M u]-6 \beta \widetilde{L}^{-1}\left[\left(\frac{d u}{d x}\right)^{2} \frac{d^{2} u}{d^{2} x}\right]
$$


For series solution we use summation on (26)

$$
\begin{aligned}
\sum_{n=0}^{\infty} u_{n}= & C_{1} x+C_{2}+m \frac{x^{2}}{2} \\
& +M \widetilde{L}^{-1}\left(\sum_{n=0}^{\infty} u_{n}\right)-6 \beta \widetilde{L}^{-1} \\
& \times\left[\frac{d}{d x}\left(\sum_{n=0}^{\infty} u_{n}\right)^{2} \frac{d^{2}}{d x^{2}}\left(\sum_{n=0}^{\infty} u_{n}\right)\right] .
\end{aligned}
$$

Adomian polynomials are defined from (27) as follows:

$$
\begin{array}{r}
\widetilde{A}_{n}=\left(\frac{d}{d x}\left(\sum_{n=0}^{\infty} u_{n}\right)^{2}\right)\left[\frac{d^{2}}{d x^{2}}\left(\sum_{n=0}^{\infty} u_{n}\right)\right], \\
n \geq 0 .
\end{array}
$$

From (28) when $n \geq 0$, the Adomian polynomials in components form are

$$
\begin{gathered}
\widetilde{A}_{0}=\left(\frac{d u_{0}}{d x}\right)^{2} \frac{d^{2} u_{0}}{d x^{2}}, \\
\widetilde{A}_{1}=\left(\frac{d u_{0}}{d x}\right)^{2} \frac{d^{2} u_{1}}{d x^{2}}+2 \frac{d u_{0}}{d x} \frac{d u_{1}}{d x} \\
\widetilde{A}_{2}=\left(\frac{d u_{0}}{d x}\right)^{2} \frac{d^{2} u_{2}}{d x^{2}}+\left(\frac{d u_{1}}{d x}\right)^{2} \frac{d^{2} u_{0}}{d x^{2}} \\
+2 \frac{d u_{0}}{d x} \frac{d u_{2}}{d x} \frac{d^{2} u_{0}}{d x^{2}} .
\end{gathered}
$$

The series solution becomes

$$
\begin{aligned}
u_{0}+u_{1}+u_{2}+\cdots= & C_{1} x+C_{2}+m \frac{x^{2}}{2} \\
& +M L^{-1}\left[u_{0}+u_{1}+u_{2}+\cdots\right] \\
& -6 \beta L^{-1}\left[\widetilde{A}_{0}+\widetilde{A}_{1}+\widetilde{A}_{2}+\cdots\right] .
\end{aligned}
$$

The velocity components are obtained by comparing both sides of (33).

\subsubsection{Zero Component Problem. Consider}

$$
u_{0}(x)=C_{1} x+C_{2}+m \frac{x^{2}}{2}
$$

From (16) the boundary condition, for $n=0$, is

$$
u_{0}(0)=\alpha-\Lambda\left(\frac{d u_{0}}{d x}+2 \beta\left(\frac{d u_{0}}{d x}\right)^{3}\right), \quad \frac{d u_{0}(1)}{d x}=0 .
$$

By making use of (32) in (31), after simplification we obtain

$$
u_{0}(x)=\alpha+\frac{m x^{2}}{2}-m x+\Lambda\left(m+2 \beta m^{3}\right) .
$$

4.1.2. First Component Problem. Consider

$$
u_{1}(x)=M L^{-1}\left[u_{0}\right]-6 \beta L^{-1}\left[\widetilde{A}_{0}\right] .
$$

From (17) the boundary condition, for $n=1$, is

$$
u_{1}(0)=-\Lambda\left(\frac{d u_{1}}{d x}+6 \beta\left(\frac{d u_{0}}{d x}\right)^{2} \frac{d u_{1}}{d x}\right), \quad \frac{d u_{1}(1)}{d x}=0 .
$$

The solution is

$$
\begin{aligned}
u_{1}(x)=[ & \left(1+6 m^{2} \beta\right) \Lambda \\
& \times\left(\frac{m M}{3}-M \alpha+2 m^{3} \beta-m M \Lambda-2 m^{3} M \beta \Lambda\right) \\
& +\left(\frac{m M}{3}-M \alpha+2 m^{3} \beta-m M \Lambda-2 m^{3} M \beta \Lambda\right) x \\
& +\left(\frac{M \alpha}{2}-3 m^{3} \beta+\frac{m M \Lambda}{2}+m^{3} M \beta \Lambda\right) x^{2} \\
& \left.+\left(2 m^{3} \beta-\frac{m M}{6}\right) x^{3}+\left(\frac{m M}{24}-\frac{m^{3} \beta}{2}\right) x^{4}\right] .
\end{aligned}
$$

4.1.3. Second Component Problem. Consider

$$
u_{2}(x)=M L^{-1}\left[u_{1}\right]-6 \beta L^{-1}\left[\widetilde{A}_{1}\right] .
$$

From (17) the boundary condition, for $n=2$, is

$$
\begin{array}{r}
u_{2}(0) \\
=-\Lambda\left(\frac{d u_{2}}{d x}\left(1+6 \beta\left(\frac{d u_{0}}{d x}\right)^{2}\right)+6 \beta\left(\frac{d u_{1}}{d x}\right)^{2} \frac{d u_{0}}{d x}\right), \\
\frac{d u_{2}(1)}{d x}=0 .
\end{array}
$$

The solution is

$$
u_{2}(x)=\Psi_{0}-\Psi_{1} x+\Psi_{2} x^{2}-\Psi_{3} x^{3}+\Psi_{4} x^{4}-\Psi_{5} x^{5}+\Psi_{6} x^{6} .
$$

The series solution up to the second component is

$$
u(x)=u_{0}(x)+u_{1}(x)+u_{2}(x) .
$$

Using (33), (36), and (39) in (40), we have

$$
u(x)=\Phi_{0}-\Phi_{1} x+\Phi_{2} x^{2}-\Phi_{3} x^{3}+\Phi_{4} x^{4}-\Phi_{5} x^{5}+\Phi_{6} x^{6}
$$

The constants $\left(\Psi_{0}-\Psi_{6}\right)$ and $\left(\Phi_{0}-\Phi_{6}\right)$ are given in the appendix.

The dimensionless shear stress is

$$
\tau_{x y}=\frac{\mu \nu}{\delta^{2}}\left[\frac{d u}{d x}+2 \beta\left(\frac{d u}{d x}\right)^{3}\right] .
$$


The shear rate becomes

$$
\begin{aligned}
\left.\tau_{x y}\right|_{x=0} & \\
=\frac{\mu \nu}{\delta^{2}}[25( & 9 m^{2} M \alpha \beta+90 m^{2} M^{2} \alpha \beta \Lambda-15 M \alpha \\
+ & 5 M^{2} \alpha+15 M^{2} \alpha \Lambda-180 m^{5} \beta^{2} \\
+ & 180 m^{2} \beta^{2} M^{2} \Lambda^{2}-15 m+5 m M \\
- & 2 m M^{2}-15 \Lambda m M \Lambda+15 m M^{2} \Lambda^{2} \\
+ & 30 m^{3} \beta-36 M m^{3} \beta+30 \Lambda M m^{3} \beta \\
& \left.-20 \Lambda M^{2} m^{3} \beta+20 \Lambda^{2} M^{2} m^{3} \beta\right) \\
+ & 90 m^{2} M \alpha \beta+90 m^{2} M^{2} \alpha \beta \Lambda-15 M \alpha \\
+ & 5 M^{2} \alpha+15 M^{2} \alpha \Lambda-180 m^{5} \beta^{2} \\
+ & 180 m^{2} \beta^{2} M^{2} \Lambda^{2}-15 m+5 m M \\
& -2 m M^{2}-15 \Lambda m M \Lambda+15 m M^{2} \Lambda^{2} \\
+ & 30 m^{3} \beta-36 M m^{3} \beta+30 \Lambda M m^{3} \beta \\
& \left.\left.-20 \Lambda M^{2} m^{3} \beta+20 \Lambda^{2} M^{2} m^{3} \beta\right)^{3}\right] .
\end{aligned}
$$

The coefficient of skin friction is defined as follows:

$$
C_{f}(0)=\frac{\left.\tau_{x y}\right|_{x=0}}{(1 / 2) \rho U^{2}}
$$

By use of (43), we have

$$
\begin{aligned}
& C_{f}(0) \\
& \qquad \begin{aligned}
=\frac{2}{R_{e}^{2}}[25( & \\
- & 15 M m^{2} M \alpha \beta+90 m^{2} M^{2} \alpha \beta \Lambda \\
- & 180 m^{5} \beta^{2}+180 m^{2} \beta^{2} M^{2} \Lambda^{2}-15 m \\
+ & 5 m M-2 m M^{2}-15 \Lambda m M \Lambda+15 m M^{2} \Lambda^{2} \\
+ & 30 m^{3} \beta-36 M m^{3} \beta+30 \Lambda M m^{3} \beta \\
& \left.-20 \Lambda M^{2} m^{3} \beta+20 \Lambda^{2} M^{2} m^{3} \beta\right) \\
+ & \left(90 m^{2} M \alpha \beta+90 m^{2} M^{2} \alpha \beta \Lambda\right. \\
- & 15 M \alpha+5 M^{2} \alpha+15 M^{2} \alpha \Lambda
\end{aligned}
\end{aligned}
$$

$$
\begin{aligned}
& -180 m^{5} \beta^{2}+180 m^{2} \beta^{2} M^{2} \Lambda^{2} \\
& -15 m+5 m M-2 m M^{2} \\
& -15 \Lambda m M \Lambda+15 m M^{2} \Lambda^{2}+30 m^{3} \beta \\
& -36 M m^{3} \beta+30 \Lambda M m^{3} \beta \\
& \left.\left.-20 \Lambda M^{2} m^{3} \beta+20 \Lambda^{2} M^{2} m^{3} \beta\right)^{3}\right] .
\end{aligned}
$$

\section{Volume Flow Rate and Average Velocity}

Volume flow rate in nondimensional form is as follows:

$$
Q=\int_{0}^{1} u(x) d x .
$$

Using velocity field from (41), volume flow rate is obtained as follows.

Average velocity $u$ in dimensionless form is given by

$$
\begin{gathered}
u=Q, \\
Q=-\frac{m}{3}+\frac{2 m M}{15}-\frac{17 m M^{2}}{315}+\alpha-\frac{M \alpha}{3} \\
+\frac{2 M^{2} \alpha}{15}+\frac{2 m^{3} \beta}{5}-\frac{64}{105} m^{3} M \beta
\end{gathered}
$$$$
+\frac{6}{5} m^{2} M \alpha \beta-\frac{12 m^{5} \beta^{2}}{7}+m \Lambda
$$$$
-\frac{1}{9} m M^{2} \Lambda-M \alpha \Lambda+\frac{2}{3} M^{2} \alpha \Lambda
$$$$
+4 m^{3} \beta \Lambda-\frac{8}{15} m^{3} M \beta \Lambda-\frac{33}{20} m^{3} M^{2} \beta \Lambda
$$$$
+\frac{15}{2} m^{2} M^{2} \alpha \beta \Lambda-6 m M^{2} \alpha^{2} \beta \Lambda
$$$$
-\frac{143}{5} m^{5} M \beta^{2} \Lambda+78 m^{4} M \alpha \beta^{2} \Lambda
$$$$
-144 m^{7} \beta^{3} \Lambda-m M \Lambda^{2}+\frac{1}{3} m M^{2} \Lambda^{2}
$$$$
+M^{2} \alpha \Lambda^{2}-4 m^{3} M \beta \Lambda^{2}+\frac{29}{6} m^{3} M^{2} \beta \Lambda^{2}
$$$$
+54 m^{5} M \beta^{2} \Lambda^{2}+12 m^{5} M^{2} \beta^{2} \Lambda^{2}
$$$$
+12 m^{4} M^{2} \alpha \beta^{2} \Lambda^{2}+84 m^{7} M \beta^{3} \Lambda^{2}
$$$$
+m M^{2} \Lambda^{3}+8 m^{3} M^{2} \beta \Lambda^{3}
$$$$
+36 m^{5} M^{2} \beta^{2} \Lambda^{3}+48 m^{7} M^{2} \beta^{3} \Lambda^{3} .
$$ 


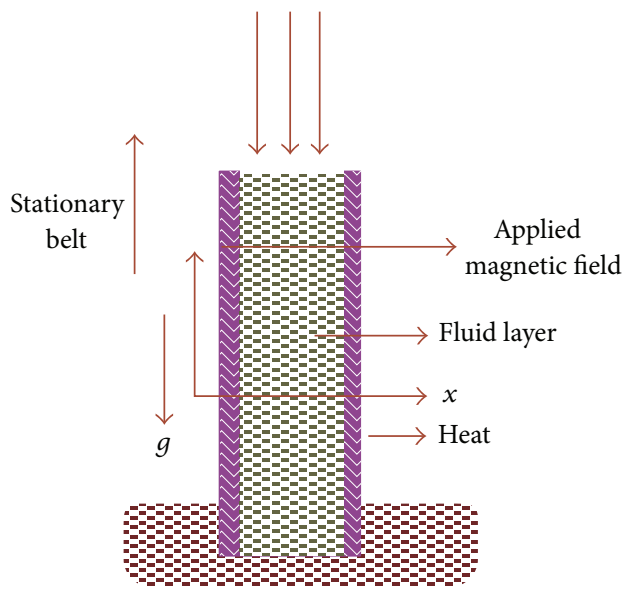

FIGURE 2: Geometry of the drainage problem.

\section{Temperature Distribution in \\ Case of Lift Problem}

On substituting the series solution for velocity field given in (41) in (15) and solving corresponding to the boundary condition given in (18) for fixed values of $\alpha=0.1 ; m=0.5$; $M=1 ; \Lambda=0.4 ; \beta=1 ; \lambda=100$, we obtained

$$
\begin{aligned}
\Theta(x)= & 7.1923 x-12.7305 x^{2}+9.0310 x^{3} \\
& -2.4798 x^{4}-0.3026 x^{5}+0.4717 x^{6} \\
& -0.2248 x^{7}+0.03702 x^{8}+0.01515 x^{9} \\
& -0.0133 x^{10}+0.0044 x^{11}-0.00032 x^{12} \\
& -0.00037 x^{13}+0.000202 x^{14}-0.000048 x^{15} \\
& +0.00000156 x^{16}+0.000003 x^{17}-0.0000014 x^{18} \\
& +3.3326 \times 10^{-7} x^{19}-5.1691 \times 10^{-8} x^{20} \\
& +5.0040 \times 10^{-9} x^{21}-2.2745 \times 10^{-10} x^{22} .
\end{aligned}
$$

\section{Formulation of Drainage Problem}

The geometry and assumptions of the problem are the same as those in the previous problem. Consider a film of nonNewtonian liquid draining at volume flow rate $Q$ down the vertical belt, as shown in Figure 2 . The belt is stationary and the fluid drain down the belt due to gravity. The coordinate system is selected in the same way as that in the previous case. Assuming the flow is steady, laminar and external pressure is neglected. Consider that fluid shear forces balance gravity and the film thickness remain constant.
Boundary condition for electrically conducting drainage problem is as follows:

$$
\begin{aligned}
& u=-\gamma T_{x y} \quad \text { at } \quad x=0, \\
& \frac{d u}{d x}=0, \quad \text { at } x=\delta .
\end{aligned}
$$

Using nondimensional variables the slip boundary conditions for drainage problem become

$$
\begin{aligned}
& u_{n}(0)=-\Lambda\left(\frac{d u_{n}}{d x}+2 \beta\left(\frac{d u_{n}}{d x}\right)^{3}\right), \quad \frac{d u_{n}(1)}{d x}=0 \text {, } \\
& n \geq 0 \text {. }
\end{aligned}
$$

7.1. Solution of the Drainage Problem by ADM. Using ADM on (14), the Adomian polynomials in (29) for both problems are the same. The different velocity components are obtained as follows.

\subsubsection{Zero Component Problem. Consider}

$$
\begin{gathered}
u_{0}(x)=C_{1}+C_{2} x-m \frac{x^{2}}{2}, \\
u_{0}(0)=-\Lambda\left(\frac{d u_{0}}{d x}+2 \beta\left(\frac{d u_{0}}{d x}\right)^{3}\right), \quad \frac{d u_{0}(1)}{d x}=0 .
\end{gathered}
$$

The solution is

$$
u_{0}(x)=m x-\frac{m x^{2}}{2}-\Lambda\left(m+2 \beta m^{3}\right)
$$

7.1.2. First Component Problem. Consider

$$
\begin{gathered}
u_{1}(x)=M L^{-1}\left[u_{0}\right]-6 \beta L^{-1}\left[\widetilde{A}_{0}\right] \\
u_{1}(0)=-\Lambda\left(\frac{d u_{1}}{d x}+6 \beta\left(\frac{d u_{0}}{d x}\right)^{2} \frac{d u_{1}}{d x}\right), \quad \frac{d u_{1}(1)}{d x}=0 .
\end{gathered}
$$

For different velocity components, the Adomian polynomials mentioned in (29) are used:

$$
\begin{aligned}
u_{1}(x)= & \left(1+6 m^{2} \beta\right) \Lambda \\
& \times\left(-\frac{m M}{3}-2 m^{3} \beta+m M \Lambda+2 m^{3} M \beta \Lambda\right) \\
& +\left(-\frac{m M}{3}-2 m^{3} \beta+m M \Lambda+2 m^{3} M \beta \Lambda\right) x \\
& +\left(3 m^{3} \beta-\frac{m M \Lambda}{2}-m^{3} M \beta \Lambda\right) x^{2} \\
& +\left(\frac{m M}{6}-2 m^{3} \beta\right) x^{3}+\left(-\frac{m M}{24}+\frac{m^{3} \beta}{2}\right) x^{4} .
\end{aligned}
$$


7.1.3. Second Component Problem. Consider

$$
u_{2}(x)=M L^{-1}\left[u_{1}\right]-6 \beta L^{-1}\left[\widetilde{A}_{1}\right],
$$

$u_{2}(0)$

$$
\begin{array}{r}
=-\Lambda\left(\frac{d u_{2}}{d x}\left(1+6 \beta\left(\frac{d u_{0}}{d x}\right)^{2}\right)+6 \beta\left(\frac{d u_{1}}{d x}\right)^{2} \frac{d u_{0}}{d x}\right), \\
\frac{d u_{2}(1)}{d x}=0 .
\end{array}
$$

The solution is

$$
u_{2}(x)=\xi_{0}-\xi_{1} x+\xi_{2} x^{2}-\xi_{3} x^{3}+\xi_{4} x^{4}-\xi_{5} x^{5}+\xi_{6} x^{6} .
$$

The series solution of the velocity field is

$$
u(x)=u_{0}(x)+u_{1}(x)+u_{2}(x) .
$$

Substituting (53), (55), and (57) in (58),

$$
u(x)=\omega_{0}-\omega_{1} x+\omega_{2} x^{2}-\omega_{3} x^{3}+\omega_{4} x^{4}-\omega_{5} x^{5}+\omega_{6} x^{6} .
$$

The constants $\left(\xi_{0}-\xi_{6}\right)$ and $\left(\omega_{0}-\omega_{6}\right)$ are defined and listed in the appendix.

Substituting (59) the shear rate is obtained as

$$
\begin{aligned}
\left.\tau_{x y}\right|_{x=0} & \begin{array}{c}
\mu \nu \\
\delta^{2}
\end{array}\left[12 m^{5} \beta^{2}-12 m^{5} \beta^{2} M^{2} \Lambda^{2}+m-\frac{m M}{3}\right. \\
& +m \Lambda M+\frac{2 M^{2} m}{15}-M^{2} \Lambda^{2} m-2 m^{3} \beta \\
& +\frac{36}{15} m^{3} M \beta-2 m^{3} M \beta \Lambda+\frac{4}{3} m^{3} M^{2} \beta \Lambda \\
& -8 m^{3} M^{2} \beta \Lambda^{2}+2 \beta \\
& +\left(12 m^{5} \beta^{2}-12 m^{5} \beta^{2} M^{2} \Lambda^{2}+m-\frac{m M}{3}\right. \\
& +m^{2} \Lambda M+\frac{2 M^{2} m}{15}-M^{2} \Lambda^{2} m \\
& -2 m^{3} \beta+\frac{36}{15} m^{3} M \beta-2 m^{3} M \beta \Lambda \\
& \left.\left.+\frac{4}{3} m^{3} M^{2} \beta \Lambda-8 m^{3} M^{2} \beta \Lambda^{2}\right)^{3}\right] .
\end{aligned}
$$

\section{Volume Flow Rate and Average Velocity of Thin Film Flow}

Using (59) in (47) we obtain the following.

The average velocity is defined as $\bar{u} / \delta$

$$
\begin{gathered}
\bar{u}=Q, \\
Q=\frac{m}{3}-\frac{2 m M}{15}+\frac{17 m M^{2}}{315}-\frac{2 m^{3} \beta}{5}
\end{gathered}
$$

$$
\begin{aligned}
& +\frac{64}{105} m^{3} M \beta+\frac{12 m^{5} \beta^{2}}{7}-m \Lambda \\
& +\frac{1}{9} m M^{2} \Lambda-4 m^{3} \beta \Lambda+\frac{8}{15} m^{3} M \beta \Lambda \\
& +\frac{33}{20} m^{3} M^{2} \beta \Lambda+\frac{143}{5} m^{5} M \beta^{2} \Lambda \\
& +144 m^{7} \beta^{3} \Lambda+m M \Lambda^{2}-\frac{1}{3} m M^{2} \Lambda^{2} \\
& +4 m^{3} M \beta \Lambda^{2}-\frac{29}{6} m^{3} M^{2} \beta \Lambda^{2} \\
& -54 m^{5} M \beta^{2} \Lambda^{2}-3 m^{5} M^{2} \beta^{2} \Lambda^{2} \\
& -84 m^{7} M \beta^{3} \Lambda^{2}-m M^{2} \Lambda^{3}-8 m^{3} M^{2} \beta \Lambda^{3} \\
& -36 m^{5} M^{2} \beta^{2} \Lambda^{3}-48 m^{7} M^{2} \beta^{3} \Lambda^{3} .
\end{aligned}
$$

\section{Heat Distribution for Drainage Problem}

Solving (15) with boundary conditions given in (18), after making use of (59) for fixed values of $m=0.5, M=1$, $\Lambda=0.4, \beta=1$, and $\lambda=100$, we obtain

$$
\begin{aligned}
\Theta(x)= & 7.7356 x-14.3046 x^{2}+11.1370 x^{3} \\
& -4.2154 x^{4}+0.57907 x^{5}+0.241003 x^{6} \\
& -0.2503 x^{7}+0.0947 x^{8}-0.01404 x^{9} \\
& -0.0068 x^{10}+0.0053 x^{11}-0.0016 x^{12} \\
& +0.000103 x^{13}+0.000127 x^{14} \\
& -0.0000609 x^{15}+0.0000126 x^{16} \\
& -8.11926 \times 10^{-8} x^{17}-7.8766446 \\
& \times 10^{-7} x^{18}+2.651929 \times 10^{-7} x^{19} \\
& -4.828779 \times 10^{-8} x^{20}+5.004021 \\
& \times 10^{-9} x^{21}-2.274555 \times 10^{-10} x^{22}
\end{aligned}
$$

\section{Results and Discussion}

The effects of magnetic parameter $M$, non-Newtonian parameter $\beta$, gravitational parameter $m$, slip parameter $\Lambda$, and the dimensionless number $\lambda$, for both lift and drainage velocity profiles, are discussed in Figures 5-14. Figures 1 and 2 show the geometry of lift and drainage velocity profiles. Figures 3 and 4 show the comparison of OHAM and ADM for lift and drainage velocity profiles. Numerical results and absolute error for both problems are shown in Tables 1 and 2 , respectively. Figures 5 and 10 show that the rise in the non-Newtonian parameter $\beta$ increases the speed of flow. For 


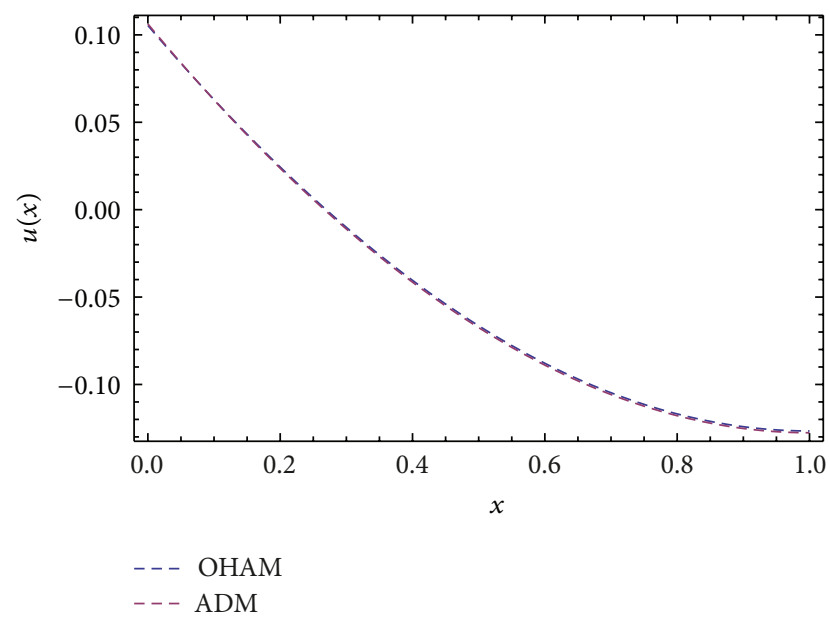

FIgURE 3: Comparison of ADM and OHAM methods for lift velocity profile is shown for the given parameters and auxiliary constants. $m=0.5, M=0.1, \beta=0.3, \Lambda=0.01, \alpha=0.1, C_{1}=-0.6221311197$, and $C_{2}=-0.0208205048$.

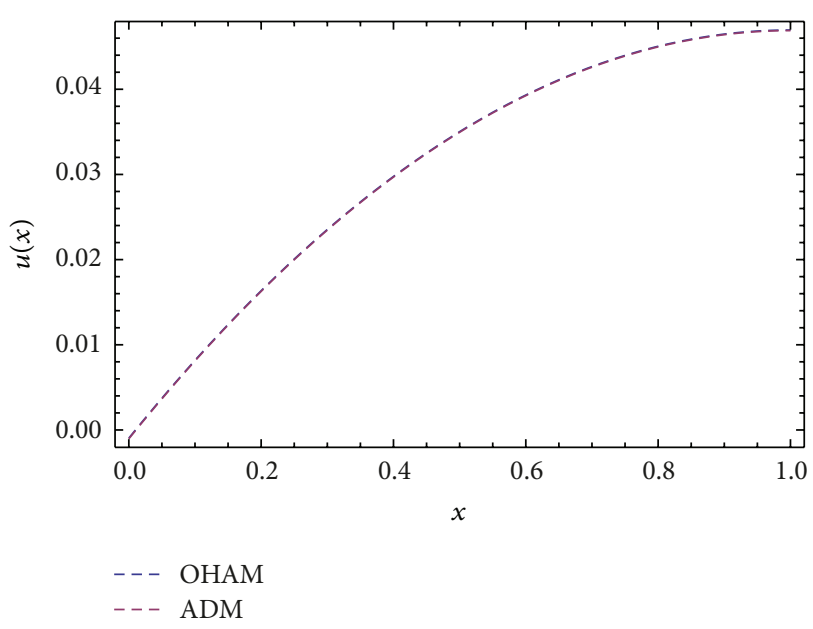

FIgURE 4: Comparison of ADM and OHAM methods for drainage velocity profile is shown for the given parameters and auxiliary constants. $m=0.1, M=0.1, \beta=0.3, \Lambda=0.01, \alpha=0.1$, $C_{1}=-0.93798569$, and $C_{2}=-0.000741729$.

small values of $\beta$, the velocity profile differs little from the Newtonian one; however when $\beta$ is increased, these profiles become more flattened showing the shear-thinning effect. Behavior of the velocity field $u$, for different values of $M$, by fixing other physical parameters, is shown in Figures 6 and 11 for lifting and drainage of fluid, respectively. Here, it can be seen that the boundary layer thickness is reciprocal to the transverse magnetic field and the velocity decreases as one progresses towards the surface of the fluid. We note that the velocity of fluid is maximum at the surface of the belt and minimum at the surface. Moreover, it is to be noted that for large values of $M$, the velocity increases rapidly as compared to small values. When the gravitational parameter $m$ increases, the velocity decreases in lifting flow and increases in case of drainage. This can be seen from

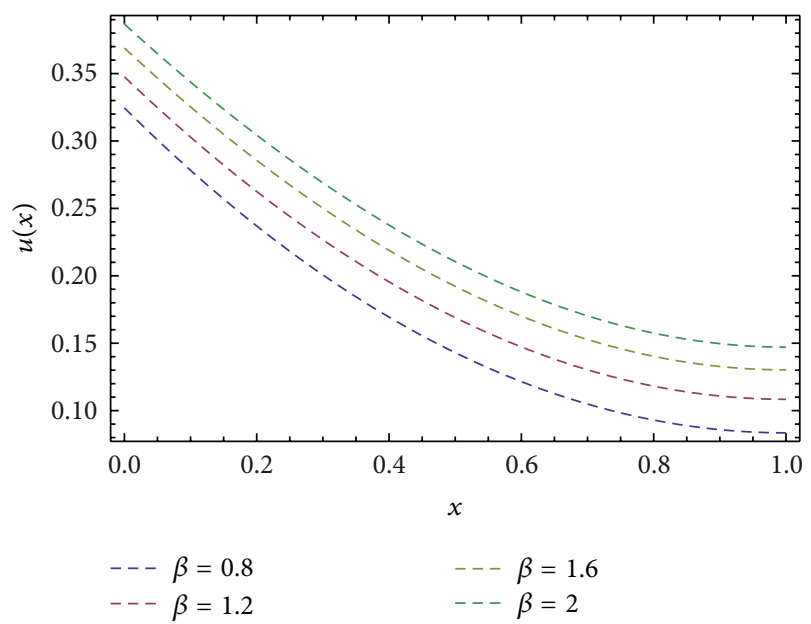

Figure 5: The influence of non-Newtonian $\beta$ on velocity profile for lifting problem keeping $\alpha=0.1, m=0.5, M=1$, and $\Lambda=0.4$.

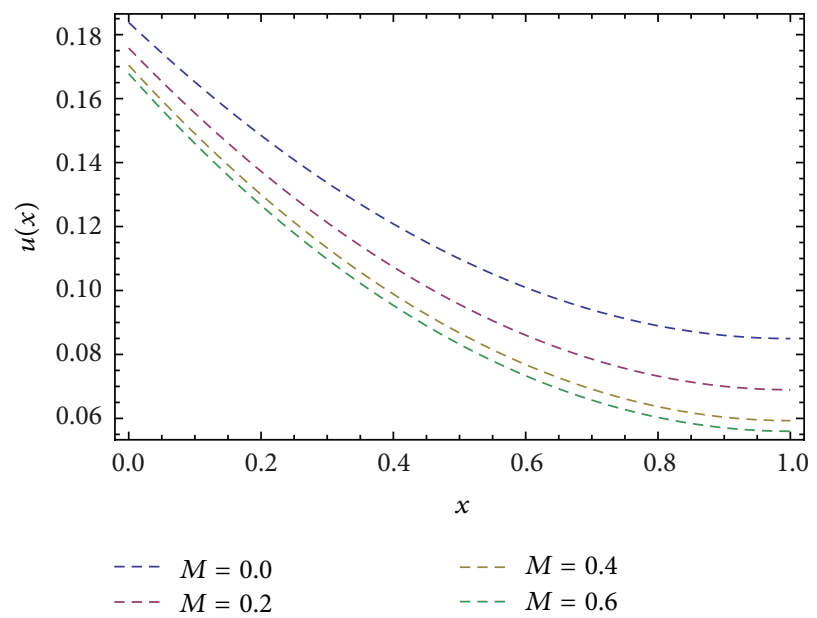

FIGURE 6: The effect of magnetic force " $M$ " on the lift velocity profile keeping other parameters fixed. $\alpha=0.1, m=0.2, \beta=1.2$, and $\Lambda=0.4$.

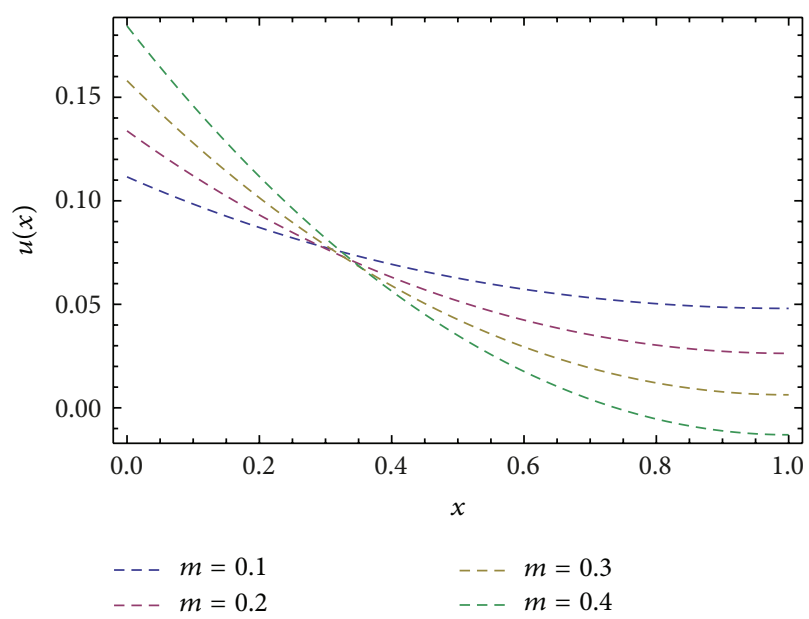

FIGURE 7: The figure shows this gravity effect of " $m$ " on velocity profile for lift problem, where $\alpha=0.1, \beta=1, M=1$, and $\Lambda=0.2$. 


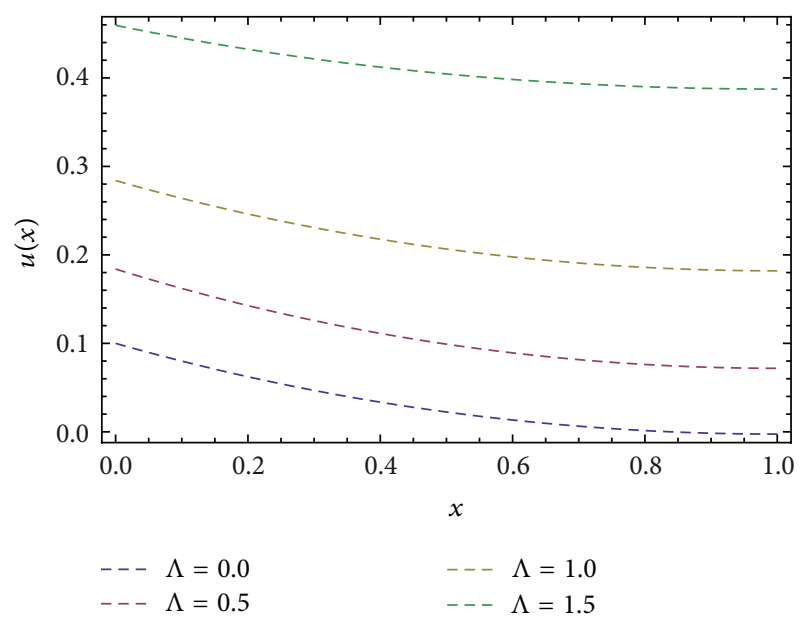

FIGURE 8: Variation of velocity for various values of slip parameter $\Lambda$, by fixing $\alpha=0.1 ; m=0.2 ; \beta=1.2 ; M=0.6$.

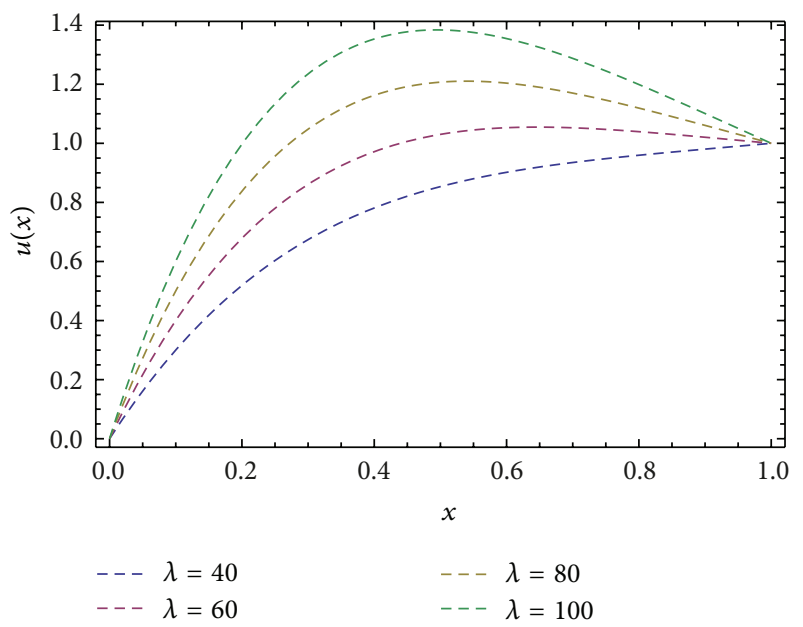

Figure 9: The influence of the dimensionless " $\lambda$ " on the temperature distribution for lift problem keeping, $\alpha=0.5, \beta=0.2, M=2$, and $m=1$.

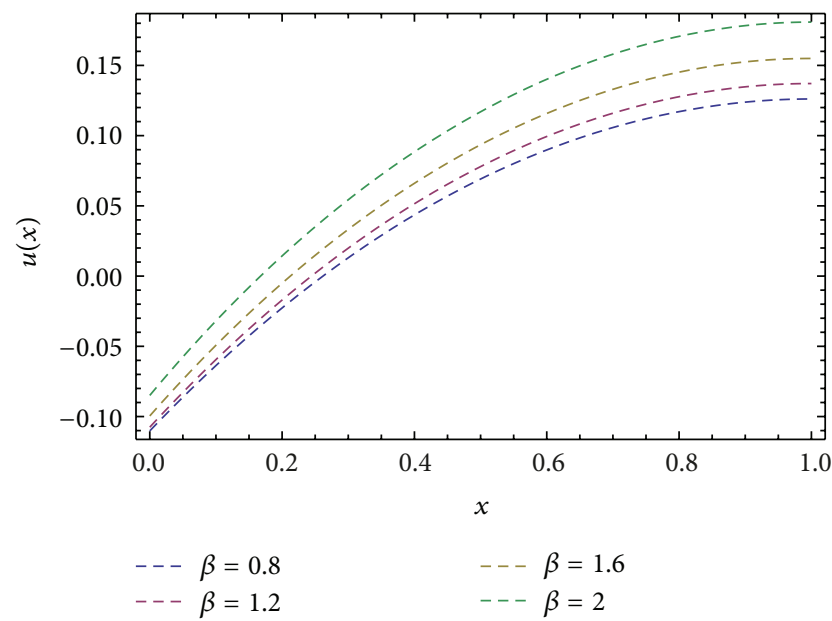

Figure 10: The influence of the non-Newtonian effect " $\beta$ " on the velocity profile $u(x)$ of drainage problem keeping $m=0.5, M=1$, and $\Lambda=0.2$.
TABLE 1: Comparison of (OHAM) and (ADM) for lift velocity profile.

\begin{tabular}{lccc}
\hline$x$ & OHAM & ADM & Absolut error \\
\hline 0.0 & 0.105386 & 0.106257 & $0.870 \times 10^{-3}$ \\
0.1 & 0.062934 & 0.062767 & $0.167 \times 10^{-3}$ \\
0.2 & 0.024379 & 0.023672 & $0.707 \times 10^{-3}$ \\
0.3 & -0.010137 & -0.011089 & $0.953 \times 10^{-3}$ \\
0.4 & -0.0404456 & -0.041497 & $0.105 \times 10^{-2}$ \\
0.5 & -0.066426 & -0.067481 & $0.106 \times 10^{-2}$ \\
0.6 & -0.087899 & -0.088944 & $0.105 \times 10^{-2}$ \\
0.7 & -0.104748 & 0.105779 & $0.104 \times 10^{-2}$ \\
0.8 & -0.116863 & -0.117886 & $0.103 \times 10^{-2}$ \\
0.9 & -0.124166 & -0.125185 & $0.102 \times 10^{-2}$ \\
\hline
\end{tabular}

TABLE 2: Comparison of (OHAM) and (ADM) for drainage velocity profile.

\begin{tabular}{lccc}
\hline$x$ & OHAM & ADM & Absolut error \\
\hline 0.0 & -0.00096249 & -0.001043 & $0.804 \times 10^{-4}$ \\
0.1 & 0.0081824 & 0.00810405 & $0.784 \times 10^{-4}$ \\
0.2 & 0.0163486 & 0.016272 & $0.767 \times 10^{-4}$ \\
0.3 & 0.0235415 & 0.0234664 & $0.752 \times 10^{-4}$ \\
0.4 & 0.0297656 & 0.0296918 & $0.739 \times 10^{-4}$ \\
0.5 & 0.0350252 & 0.0349525 & $0.728 \times 10^{-4}$ \\
0.6 & 0.0393238 & 0.0392519 & $0.719 \times 10^{-4}$ \\
0.7 & 0.0426641 & 0.0425929 & $0.713 \times 10^{-4}$ \\
0.8 & 0.0450485 & 0.0449777 & $0.708 \times 10^{-4}$ \\
0.9 & 0.0464684 & 0.0464079 & $0.705 \times 10^{-4}$ \\
\hline
\end{tabular}

Figures 7 and 12, respectively. Due to friction force the gravitational effect seems to be smaller near the belt. It can be seen that there is a point in the domain, where the velocity of the fluid becomes approximately the same for different values of gravitational parameter. The reason is that the friction of the belt becomes negligible at this point. On increasing $m$ after this point in lifting flow, the velocity decreases due to negligible friction but in drainage flow after this point, the velocity of the fluid increases. The effect of slip parameter can be observed from Figures 8 and 13. It is noticed that the speed of the fluid near the belt is greater than the speed at the surface. When we increase the slip parameter, the velocity of the fluid increases and comparatively this increase can be seen more clearly between $\Lambda=1.0$ and $\Lambda=1.5$ because the friction goes on decreasing. Figures 9 and 14 indicate the dimensionless temperature distribution for different values of $\lambda$. It can be seen that temperature distribution increases as the dimensionless parameter $\lambda$ increases and becomes more flattened for large values of $\lambda$. Figure 15 shows the effect of local Reynolds number versus skin friction. This figure shows that the Reynolds number decreases the skin friction. For large values of Reynolds number, the skin friction vanishes. Figure 16 shows the effect of non-Newtonian parameter $\beta$ versus skin friction. 


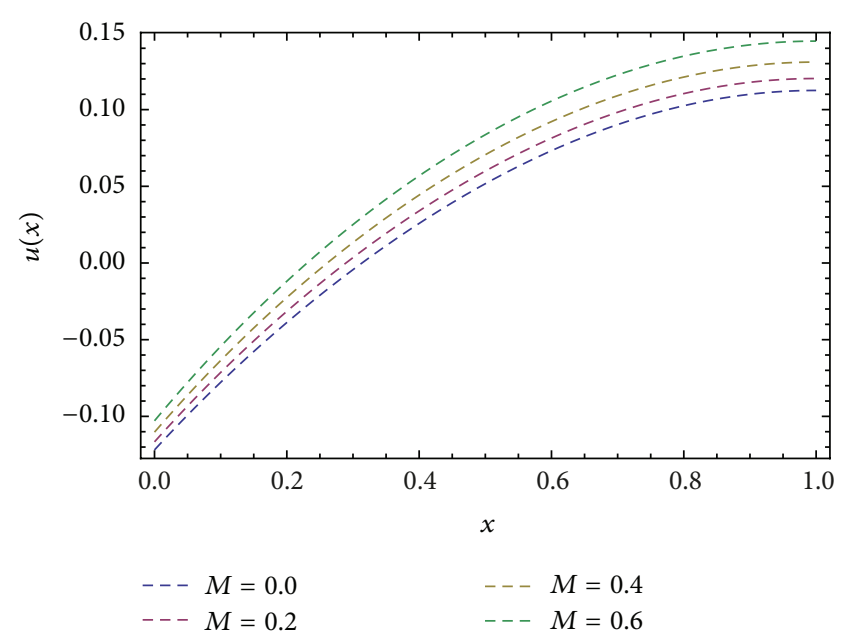

FIGURE 11: The influence of the magnetic force " $M$ " on velocity profile shown in this figure for the drainage problem, where $m=0.5$, $\beta=1$, and $\Lambda=0.2$.

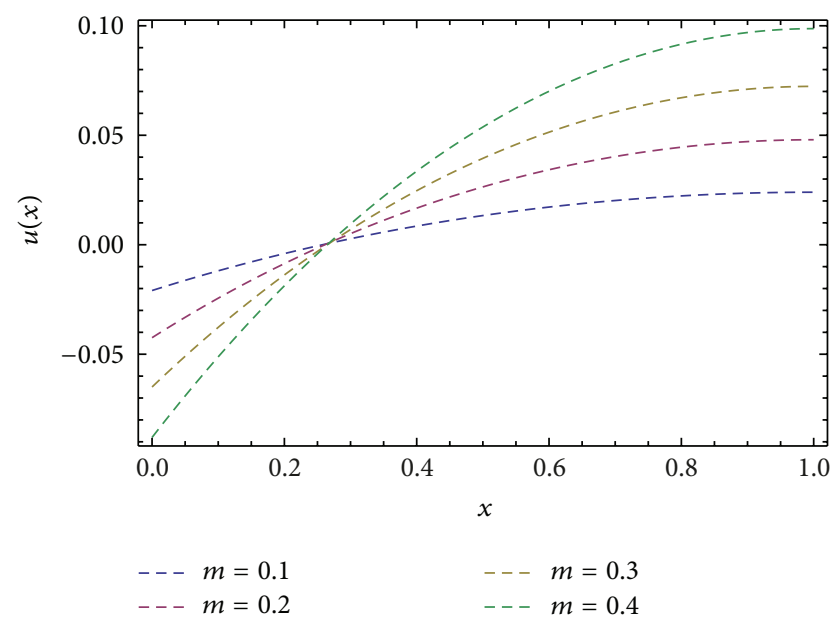

FIGURE 12: This figure shows the influence of the gravitational parameter $m$ on the velocity profile " $u(x)$ " for drainage problem keeping $\beta=1, M=1$, and $\Lambda=0.2$.

\section{Conclusion}

The constitutive equation governing the flow of a third grade fluid for lifting and drainage of fluid with slip boundary conditions is solved analytically by using Adomian decomposition method. Expression for velocity field, volume flow rate, skin friction, and temperature distribution is derived and sketched. It is concluded that velocity increases as the gravitational parameter $m$ decreases in lifting case while velocity increases as this parameter increases in drainage. For small values of $\beta$, the velocity profile differs little from the Newtonian one; however when $\beta$ is increased, these profiles become more flattened showing the shear-thinning effect. It can be seen that the boundary layer thickness is reciprocal to the transverse magnetic effect and the velocity decreases as it progresses towards the surface of the fluid. On increasing the slip parameter, the velocity of the fluid increases and

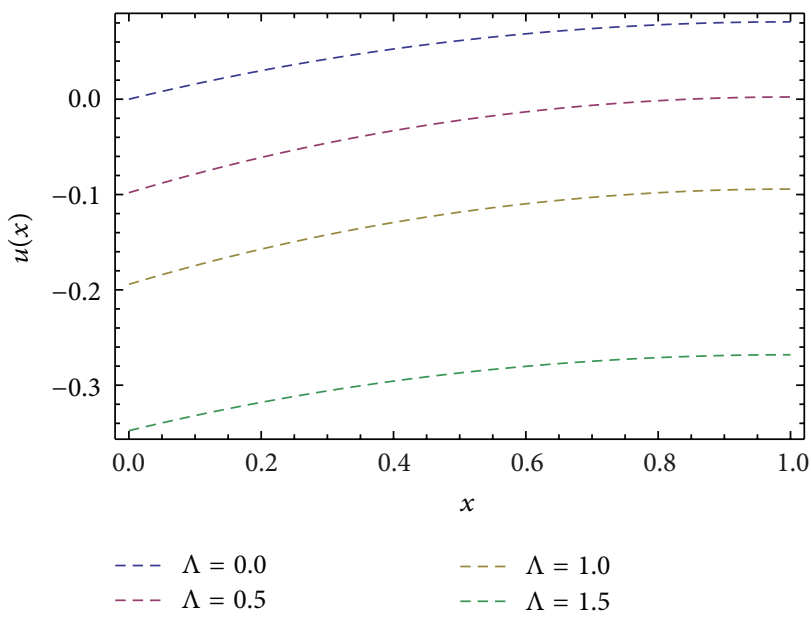

FIGURE 13: Variation of velocity for various values of slip parameter $\Lambda$, by fixing $M=0.6, m=0.2$, and $\beta=1.2$.

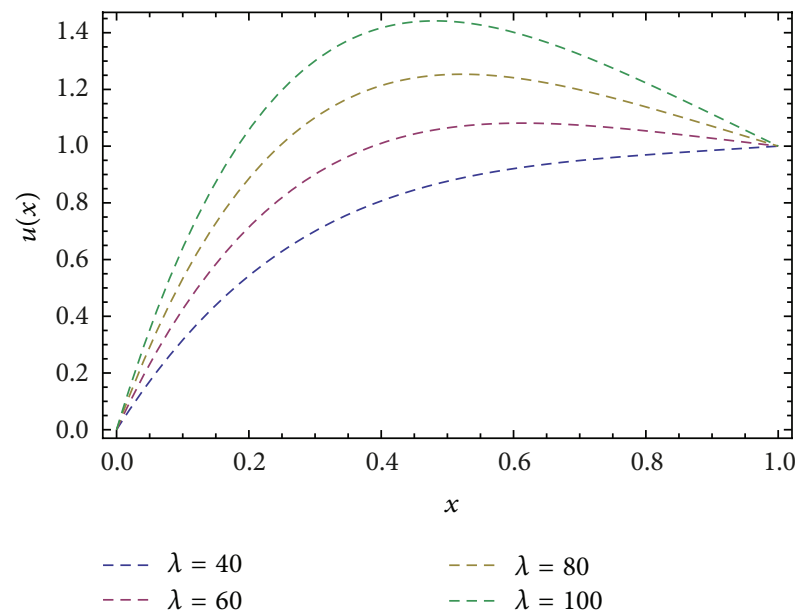

Figure 14: The influence of dimensionless number " $\lambda$ " on the temperature distribution $u(x)$, for drainage problem keeping $m=$ $0.5, M=1, \Lambda=0.4$, and $\beta=1$.

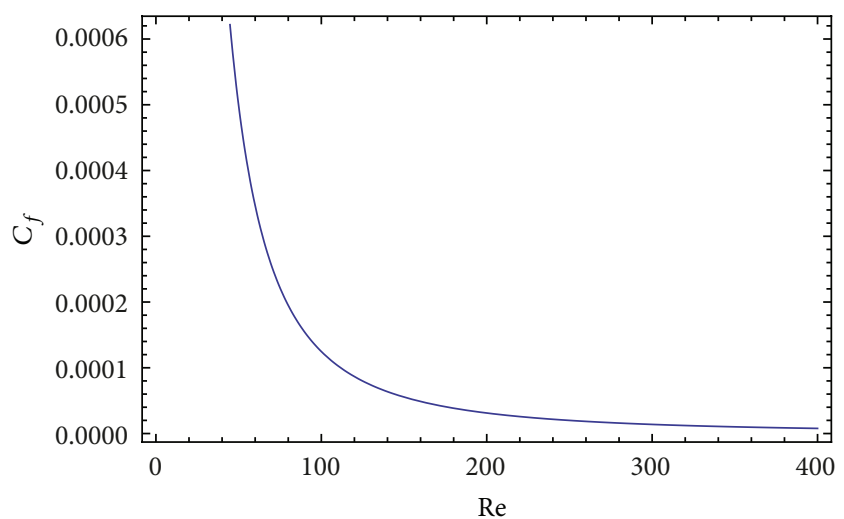

FIGURE 15: Variation of skin friction versus Reynolds number. 


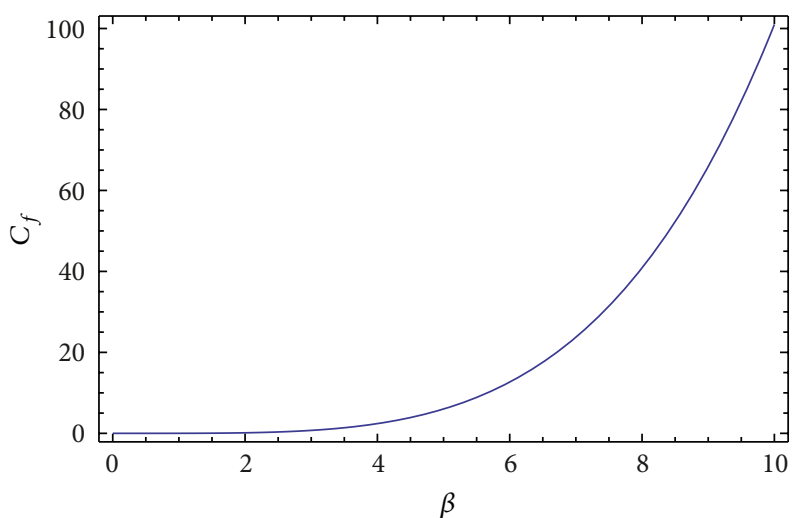

FIGURE 16: Variation of skin friction versus non-Newtonian parameter $\beta$.

comparatively this increase can be seen more clearly between $\Lambda=1.0$ and $\Lambda=1.5$ because the friction goes on decreasing. According to the best of our knowledge there is no previous literature about discussed problem; this is our first attempt to handle this problem with slip boundary condition. Also this problem is more general when compared to linear viscous model and second grade fluid model.

\section{Appendix}

$$
\begin{aligned}
& \Psi_{0}=-\frac{2}{15} \Lambda m M^{2}+\Lambda^{3} m M^{2}+\frac{1}{3} \Lambda M^{2} \alpha \\
&+\Lambda^{2} M^{2} \alpha-\frac{12}{5} \Lambda m^{3} M \beta+4 \Lambda^{2} m^{3} M \beta \\
&-\frac{5}{4} \Lambda m^{3} M^{2} \beta+\frac{13}{6} \Lambda^{2} m^{3} M^{2} \beta \\
&+8 \Lambda^{3} m^{3} M^{2} \beta+6 \Lambda m^{2} M \alpha \beta \\
&+\frac{11}{2} \Lambda m^{2} M^{2} \alpha \beta-6 \Lambda m M^{2} \alpha^{2} \beta \\
&-12 \Lambda m^{5} \beta^{2}-27 \Lambda m^{5} M \beta^{2} \\
&+66 \Lambda^{2} m^{5} M \beta^{2}-\Lambda^{2} m^{5} M^{2} \beta^{2} \\
&+36 \Lambda^{3} m^{5} M^{2} \beta^{2}+78 \Lambda m^{4} M \alpha \beta^{2} \\
&+12 \Lambda^{2} m^{4} M^{2} \alpha \beta^{2}-144 \Lambda m^{7} \beta^{3} \\
&+42 \Lambda^{2} m^{7} M \beta^{3}+48 \Lambda^{3} m^{7} M^{2} \beta^{3}, \\
&+\frac{2 m M^{2}}{15}-\Lambda^{2} m M^{2}-\frac{M^{2} \alpha}{3}-\Lambda M^{2} \alpha \\
&+\frac{12}{5} m^{3} M \beta-4 \Lambda m^{3} M \beta \\
& \Psi_{1} M^{2} \beta-8 \Lambda^{2} m^{3} M^{2} \beta \\
&+15
\end{aligned}
$$

$$
\begin{aligned}
& -6 m^{2} M \alpha \beta-6 \Lambda m^{2} M^{2} \alpha \beta \\
& +12 m^{5} \beta^{2}-12 \Lambda^{2} m^{5} M^{2} \beta^{2}
\end{aligned}
$$$$
\Psi_{2}=\frac{1}{6} \Lambda m M^{2}-\frac{1}{2} \Lambda^{2} m M^{2}
$$$$
-\frac{1}{2} \Lambda M^{2} \alpha+2 m^{3} M \beta
$$$$
-8 \Lambda m^{3} M \beta+\Lambda m^{3} M^{2} \beta
$$$$
-4 \Lambda^{2} m^{3} M^{2} \beta-9 m^{2} M \alpha \beta
$$$$
-3 \Lambda m^{2} M^{2} \alpha \beta+30 m^{5} \beta^{2}
$$$$
-12 \Lambda m^{5} M \beta^{2}-6 \Lambda^{2} m^{5} M^{2} \beta^{2},
$$

$$
\begin{aligned}
\Psi_{3}= & -\frac{m M^{2}}{18}+\frac{1}{6} \Lambda m M^{2} \\
& +\frac{M^{2} \alpha}{6}-\frac{2}{3} m^{3} M \beta \\
& -6 \Lambda m^{3} M \beta+\frac{1}{3} \Lambda m^{3} M^{2} \beta \\
& -6 m^{2} M \alpha \beta+40 m^{5} \beta^{2} \\
& -12 \Lambda m^{5} M \beta^{2},
\end{aligned}
$$

$$
\begin{aligned}
\Psi_{4}= & \frac{1}{24} \Lambda m M^{2}+\frac{M^{2} \alpha}{24}-2 m^{3} M \beta \\
& -\frac{3}{2} \Lambda m^{3} M \beta+\frac{1}{12} \Lambda m^{3} M^{2} \beta \\
& -\frac{3}{2} m^{2} M \alpha \beta+30 m^{5} \beta^{2} \\
& -3 \Lambda m^{5} M \beta^{2}, \\
\Psi_{5}= & \frac{m M^{2}}{120}-\frac{11}{10} m^{3} M \beta+12 m^{5} \beta^{2}, \\
\Psi_{6}= & \frac{m M^{2}}{720}-\frac{11}{60} m^{3} M \beta+2 m^{5} \beta^{2},
\end{aligned}
$$$$
\Phi_{0}=\Lambda m+\frac{\Lambda m M}{3}-\Lambda^{2} m M-\frac{2}{15} \Lambda m M^{2}
$$$$
+\Lambda^{3} m M^{2}+\alpha-\Lambda M \alpha+\frac{1}{3} \Lambda M^{2} \alpha
$$$$
+\Lambda^{2} M^{2} \alpha+4 \Lambda m^{3} \beta-\frac{2}{5} \Lambda m^{3} M \beta
$$$$
-4 \Lambda^{2} m^{3} M \beta-\frac{5}{4} \Lambda m^{3} M^{2} \beta
$$$$
+\frac{13}{6} \Lambda^{2} m^{3} M^{2} \beta+8 \Lambda^{3} m^{3} M^{2} \beta
$$$$
+\frac{3}{2} \Lambda m^{2} M^{2} \alpha \beta+12 \Lambda^{2} m^{2} M^{2} \alpha \beta
$$ 


$$
\begin{aligned}
& -27 \Lambda m^{5} M \beta^{2}+54 \Lambda^{2} m^{5} M \beta^{2} \\
& -\Lambda^{2} m^{5} M^{2} \beta^{2}+36 \Lambda^{3} m^{5} M^{2} \beta^{2} \\
& +8 \Lambda m^{2} M \alpha \beta^{2}-24 \Lambda^{2} m^{2} M \alpha \beta^{2} \\
& +54 \Lambda m^{4} M \alpha \beta^{2}+36 \Lambda^{2} m^{4} M^{2} \alpha \beta^{2} \\
& -144 \Lambda m^{7} \beta^{3}+84 \Lambda^{2} m^{7} M \beta^{3} \\
& +48 \Lambda^{3} m^{7} M^{2} \beta^{3}+48 \Lambda m^{4} \alpha \beta^{3} \\
& -48 \Lambda^{2} m^{4} M \alpha \beta^{3}-24 \Lambda m \alpha^{2} \beta^{3} \\
& \Phi_{1}=m-\frac{m M}{3}+\Lambda m M+\frac{2 m M^{2}}{15} \\
& -\Lambda^{2} m M^{2}+M \alpha-\frac{M^{2} \alpha}{3} \\
& -\Lambda M^{2} \alpha-2 m^{3} \beta+\frac{12}{5} m^{3} M \beta \\
& -2 \Lambda m^{3} M \beta+\frac{4}{3} \Lambda m^{3} M^{2} \beta \\
& -8 \Lambda^{2} m^{3} M^{2} \beta-6 m^{2} M \alpha \beta \\
& -6 \Lambda m^{2} M^{2} \alpha \beta+12 m^{5} \beta^{2} \\
& -12 \Lambda^{2} m^{5} M^{2} \beta^{2} \\
& \Phi_{2}=\frac{m}{2}+\frac{\Lambda m M}{2}+\frac{1}{6} \Lambda m M^{2} \\
& -\frac{1}{2} \Lambda^{2} m M^{2}+\frac{M \alpha}{2}-\frac{1}{2} \Lambda M^{2} \alpha \\
& -3 m^{3} \beta+2 m^{3} M \beta \\
& -7 \Lambda m^{3} M \beta+\Lambda m^{3} M^{2} \beta \\
& -4 \Lambda^{2} m^{3} M^{2} \beta-9 m^{2} M \alpha \beta \\
& -3 \Lambda m^{2} M^{2} \alpha \beta+30 m^{5} \beta^{2} \\
& -12 \Lambda m^{5} M \beta^{2}-3 \Lambda^{2} m^{5} M^{2} \beta^{2}, \\
& \Phi_{3}=\frac{m M}{6}-\frac{m M^{2}}{18}+\frac{1}{6} \Lambda m M^{2} \\
& +\frac{M^{2} \alpha}{6}-2 m^{3} \beta-\frac{2}{3} m^{3} M \beta \\
& -6 \Lambda m^{3} M \beta+\frac{1}{3} \Lambda m^{3} M^{2} \beta \\
& -6 m^{2} M \alpha \beta+40 m^{5} \beta^{2} \\
& -12 \Lambda m^{5} M \beta^{2}
\end{aligned}
$$

$$
\begin{aligned}
& \Phi_{4}=\frac{m M}{24}+\frac{1}{24} \Lambda m M^{2}+\frac{M^{2} \alpha}{24} \\
& -\frac{m^{3} \beta}{2}-2 m^{3} M \beta-\frac{3}{2} \Lambda m^{3} M \beta \\
& +\frac{1}{12} \Lambda m^{3} M^{2} \beta-\frac{3}{2} m^{2} M \alpha \beta \\
& +30 m^{5} \beta^{2}-3 \Lambda m^{5} M \beta^{2}, \\
& \Phi_{5}=\frac{m M^{2}}{120}-\frac{11}{10} m^{3} M \beta+12 m^{5} \beta^{2}, \\
& \Phi_{6}=\frac{m M^{2}}{720}-\frac{11}{60} m^{3} M \beta+2 m^{5} \beta^{2} \text {, } \\
& \xi_{0}=\frac{2}{15} m M^{2} \Lambda+\frac{12}{5} m^{3} M \beta \Lambda \\
& +\frac{5}{4} m^{3} M^{2} \beta \Lambda+12 m^{5} \beta^{2} \Lambda \\
& +27 m^{5} M \beta^{2} \Lambda+144 m^{7} \beta^{3} \Lambda \\
& -4 m^{3} M \beta \Lambda^{2}-\frac{13}{6} m^{3} M^{2} \beta \Lambda^{2} \\
& -66 m^{5} M \beta^{2} \Lambda^{2}+m^{5} M^{2} \beta^{2} \Lambda^{2} \\
& -84 m^{7} M \beta^{3} \Lambda^{2}-m M^{2} \Lambda^{3} \\
& -8 m^{3} M^{2} \beta \Lambda^{3}-36 m^{5} M^{2} \beta^{2} \Lambda^{3} \\
& -48 m^{7} M^{2} \beta^{3} \Lambda^{3}
\end{aligned}
$$$$
\xi_{1}=m M^{2} \Lambda^{2}-\frac{2 m M^{2}}{15}
$$$$
-\frac{12}{5} m^{3} M \beta-12 m^{5} \beta^{2}
$$$$
+4 m^{3} M \beta \Lambda-\frac{4}{3} m^{3} M^{2} \beta \Lambda
$$$$
+8 m^{3} M^{2} \beta \Lambda^{2}+12 m^{5} M^{2} \beta^{2} \Lambda^{2},
$$$$
\xi_{2}=6 m^{5} M^{2} \beta^{2} \Lambda^{2}-2 m^{3} M \beta
$$$$
-30 m^{5} \beta^{2}-\frac{1}{6} m M^{2} \Lambda
$$$$
+8 m^{3} M \beta \Lambda-m^{3} M^{2} \beta \Lambda
$$$$
+12 m^{5} M \beta^{2} \Lambda+\frac{1}{2} m M^{2} \Lambda^{2}
$$$$
+4 m^{3} M^{2} \beta \Lambda^{2}
$$ 


$$
\begin{aligned}
& \xi_{3}=\frac{m M^{2}}{18}+\frac{2}{3} m^{3} M \beta-40 m^{5} \beta^{2} \\
& -\frac{1}{6} m M^{2} \Lambda+6 m^{3} M \beta \Lambda \\
& -\frac{1}{3} m^{3} M^{2} \beta \Lambda+12 m^{5} M \beta^{2} \Lambda, \\
& \xi_{4}=m^{3} M \beta-30 m^{5} \beta^{2} \\
& -\frac{1}{24} m M^{2} \Lambda+\frac{3}{2} m^{3} M \beta \Lambda \\
& -\frac{1}{12} m^{3} M^{2} \beta \Lambda+3 m^{5} M \beta^{2} \Lambda, \\
& \xi_{5}=\frac{11}{10} m^{3} M \beta-12 m^{5} \beta^{2}-\frac{m M^{2}}{120}, \\
& \xi_{6}=-\frac{m M^{2}}{720}+\frac{11}{120} m^{3} M \beta-2 m^{5} \beta^{2}, \\
& \omega_{0}=-m \Lambda-\frac{m M \Lambda}{3}+\frac{2}{15} m M^{2} \Lambda \\
& -4 m^{3} \beta \Lambda+\frac{2}{5} m^{3} M \beta \Lambda+\frac{5}{4} m^{3} M^{2} \beta \Lambda \\
& +27 m^{5} M \beta^{2} \Lambda+144 m^{7} \beta^{3} \Lambda \\
& +m M \Lambda^{2}+4 m^{3} M \beta \Lambda^{2} \\
& -\frac{13}{6} m^{3} M^{2} \beta \Lambda^{2}-54 m^{5} M \beta^{2} \Lambda^{2} \\
& +m^{5} M^{2} \beta^{2} \Lambda^{2}-84 m^{7} M \beta^{3} \Lambda^{2} \\
& -m M^{2} \Lambda^{3}-8 m^{3} M^{2} \beta \Lambda^{3} \\
& -36 m^{5} M^{2} \beta^{2} \Lambda^{3}-48 m^{7} M^{2} \beta^{3} \Lambda^{3}, \\
& \omega_{1}=\frac{m M}{3}-m-\frac{2 m M^{2}}{15}+2 m^{3} \beta \\
& -\frac{12}{5} m^{3} M \beta-12 m^{5} \beta^{2}-m M \Lambda \\
& +2 m^{3} M \beta \Lambda-\frac{4}{3} m^{3} M^{2} \beta \Lambda \\
& +m M^{2} \Lambda^{2}+8 m^{3} M^{2} \beta \Lambda^{2} \\
& +12 m^{5} M^{2} \beta^{2} \Lambda^{2}, \\
& \omega_{2}=-\frac{m}{2}+3 m^{3} \beta-2 m^{3} M \beta \\
& -30 m^{5} \beta^{2}-\frac{m M \Lambda}{2}-\frac{1}{6} m M^{2} \Lambda \\
& +7 m^{3} M \beta \Lambda-m^{3} M^{2} \beta \Lambda
\end{aligned}
$$

$$
\begin{aligned}
& +12 m^{5} M \beta^{2} \Lambda+\frac{1}{2} m M^{2} \Lambda^{2} \\
& +4 m^{3} M^{2} \beta \Lambda^{2}+6 m^{5} M^{2} \beta^{2} \Lambda^{2}, \\
& \omega_{3}=\frac{m M^{2}}{18}-\frac{m M}{6}+2 m^{3} \beta \\
& +\frac{2}{3} m^{3} M \beta-40 m^{5} \beta^{2} \\
& -\frac{1}{6} m M^{2} \Lambda+6 m^{3} M \beta \Lambda \\
& -\frac{1}{3} m^{3} M^{2} \beta \Lambda+12 m^{5} M \beta^{2} \Lambda, \\
& \omega_{4}=-\frac{m M}{24}+\frac{m^{3} \beta}{2}+2 m^{3} M \beta \\
& -30 m^{5} \beta^{2}-\frac{1}{24} m M^{2} \Lambda \\
& +\frac{3}{2} m^{3} M \beta \Lambda-\frac{1}{12} m^{3} M^{2} \beta \Lambda \\
& +3 m^{5} M \beta^{2} \Lambda \text {, } \\
& \omega_{5}=\frac{11}{10} m^{3} M \beta-\frac{m M^{2}}{120}-12 m^{5} \beta^{2}, \\
& \omega_{6}=\frac{11}{60} m^{3} M \beta-\frac{m M^{2}}{720}-2 m^{5} \beta^{2} \text {. }
\end{aligned}
$$

\section{References}

[1] N. V. Lavrik, C. A. Tipple, M. J. Sepaniak, and P. G. Datskos, "Gold nano-structures for transduction of biomolecular interactions into micrometer scale movements," Biomedical Microdevices, vol. 3, no. 1, pp. 35-44, 2001.

[2] A. R. A. Khaled and K. Vafai, "Hydromagnetic squeezed flow and heat transfer over a sensor surface," International Journal of Engineering Science, vol. 42, pp. 509-519, 2004.

[3] S. Nadeem and M. Awais, "Thin film flow of an unsteady shrinking sheet through porous medium with variable viscosity," Physics Letters A, vol. 372, no. 30, pp. 4965-4972, 2008.

[4] B. R. Munson and D. F. Young, Fundamentals of Fluid Mechanics, John Wiley \& Sons, New York, NY, USA, 2nd edition, 1994.

[5] M. K. Alam, A. M. Siddiqui, M. T. Rahim, and S. Islam, "Thinfilm flow of magnetohydrodynamic (MHD) Johnson-Segalman fluid on vertical surfaces using the Adomian decomposition method," Applied Mathematics and Computation, vol. 219, pp. 3956-3974, 2012.

[6] M. Hameed and R. Ellahi, "Thin film flow of non-Newtonian MHD fluid on a vertically moving belt," International Journal for Numerical Methods in Fluids, vol. 66, no. 11, pp. 1409-1419, 2011.

[7] Y. M. Aiyesimi and G. T. Okedao, "MHD flow of a third grade fluid with heat transfer down an inclined plane," Mathematical Theory and Modeling, vol. 2, no. 9, pp. 108-119, 2012. 
[8] N. Khan and T. Mahmood, "The influence of slip condition on the thin film flow of a third order fluid," International Journal of Nonlinear Science, vol. 13, no. 1, pp. 105-116, 2012.

[9] A. H. Nayfeh, Perturbation Methods, John Wiley \& Sons, New York, NY, USA, 1973.

[10] N. Jamshidi and D. D. Ganji, "Application of energy balance method and variational iteration method to an oscillation of a mass attached to a stretched elastic wire," Current Applied Physics, vol. 10, no. 2, pp. 484-486, 2010.

[11] D. D. Ganji, "A semi-analytical Technique for non-linear setting particle equation of motion original," Journal of HydroEnvironment Research, vol. 6, no. 4, pp. 323-327, 2012.

[12] M. Jalaal, D. D. Ganji, and G. Ahmad, "An analytical study on settling of non-spherical particles," Asia-Pacific Journal of Chemical Engineering, vol. 7, no. 1, pp. 63-72, 2012.

[13] M. Rafei, H. Daniali, and D. D. Ganji, "Variational iteration method for solving the epidemic model and the prey and predator problem," Applied Mathematics and Computation, vol. 186, no. 2, pp. 1701-1709, 2007.

[14] M. Jalaal and D. D. Ganji, "An analytical study on motion of a sphere rolling down an inclined plane submerged in a Newtonian fluid," Powder Technology, vol. 198, no. 1, pp. 82-92, 2010.

[15] M. Omidvar, A. Barari, M. Momeni, and D. Ganji, "New class of solutions for water infiltration problems in unsaturated soils," Geomechanics and Geoengineering, vol. 5, no. 2, pp. 127-135, 2010.

[16] A. M. Siddiqui, R. Mahmood, and Q. K. Ghori, "Homotopy perturbation method for thin film flow of a third grade fluid down an inclined plane," Chaos, Solitons and Fractals, vol. 35, no. 1, pp. 140-147, 2008.

[17] M. Jalaal, D. D. Ganji, and G. Ahmadi, "Analytical investigation on acceleration motion of a vertically falling spherical particle in incompressible Newtonian media," Advanced Powder Technology, vol. 21, no. 3, pp. 298-304, 2010.

[18] M. Jalaal and D. D. Ganji, "On unsteady rolling motion of spheres in inclined tubes filled with incompressible Newtonian fluids," Advanced Powder Technology, vol. 22, no. 1, pp. 58-67, 2011.

[19] M. Esmaeilpour and D. D. Ganji, “Application of He's homotopy perturbation method to boundary layer flow and convection heat transfer over a flat plate," Physics Letters A, vol. 372, no. 1, pp. 33-38, 2007.

[20] D. G. Domairry, A. Mohsenzadeh, and M. Famouri, "The application of homotopy analysis method to solve nonlinear differential equation governing Jeffery-Hamel flow," Communications in Nonlinear Science and Numerical Simulation, vol. 14, no. 1, pp. 85-95, 2009.

[21] A. R. Ghotbi, H. Bararnia, G. Domairry, and A. Barari, "Investigation of a powerful analytical method into natural convection boundary layer flow," Communications in Nonlinear Science and Numerical Simulation, vol. 14, no. 5, pp. 2222-2228, 2009.

[22] S. J. Liao, Beyond Perturbation: Introduction to Homotopy Analysis Method, Chapman \& Hall/CRC, Boca Raton, Fla, USA, 2003.

[23] V. Marinca, N. Herişanu, and I. Nemeş, “Optimal homotopy asymptotic method with application to thin film flow," Central European Journal of Physics, vol. 6, no. 3, pp. 648-653, 2008.

[24] N. Herișanu and V. Marinca, "Explicit analytical approximation to large-amplitude non-linear oscillations of a uniform cantilever beam carrying an intermediate lumped mass and rotary inertia," Meccanica, vol. 45, no. 6, pp. 847-855, 2010.
[25] A. M. Siddiqui, M. Ahmed, and Q. K. Ghori, “Thin film flow of non-Newtonian fluids on a moving belt," Chaos, Solitons and Fractals, vol. 33, no. 3, pp. 1006-1016, 2007.

[26] A. M. Siddiqui, R. Mahmood, and Q. K. Ghori, "Homotopy perturbation method for thin film flow of a fourth grade fluid down a vertical cylinder," Physics Letters A, vol. 352, no. 4-5, pp. 404-410, 2006.

[27] S. Chakraborty and S. K. Som, "Heat transfer in an evaporating thin liquid film moving slowly along the walls of an inclined microchannel," International Journal of Heat and Mass Transfer, vol. 48, no. 13, pp. 2801-2805, 2005.

[28] G. Adomian, Solving Frontier Problems of Physics: the Decomposition Method, Kluwer Academic Publishers, 1994.

[29] G. Adomian, "A review of the decomposition method and some recent results for nonlinear equations," Mathematical and Computer Modelling, vol. 13, no. 7, pp. 17-43, 1990.

[30] A.-M. Wazwaz, "Adomian decomposition method for a reliable treatment of the Bratu-type equations," Applied Mathematics and Computation, vol. 166, no. 3, pp. 652-663, 2005.

[31] A.-M. Wazwaz, "Adomian decomposition method for a reliable treatment of the Emden-Fowler equation," Applied Mathematics and Computation, vol. 161, no. 2, pp. 543-560, 2005. 


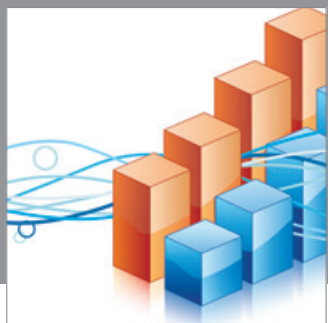

Advances in

Operations Research

mansans

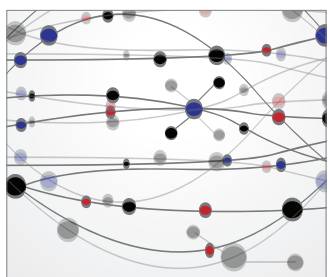

The Scientific World Journal
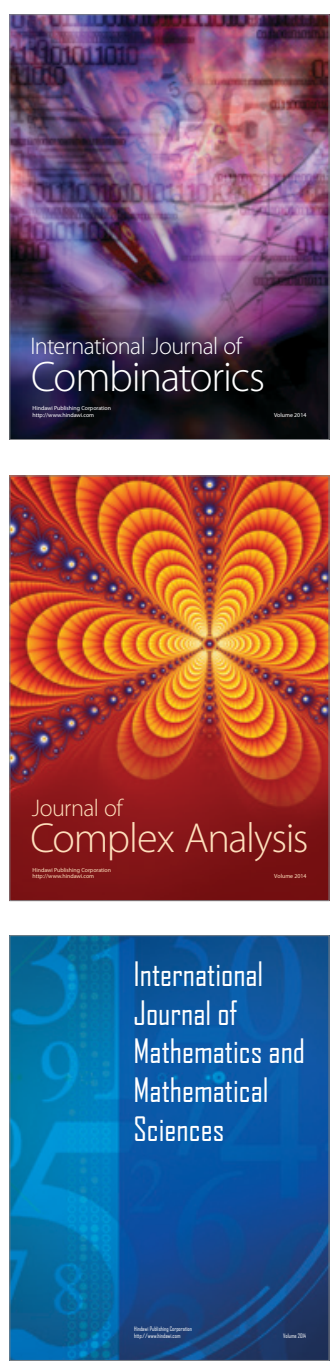
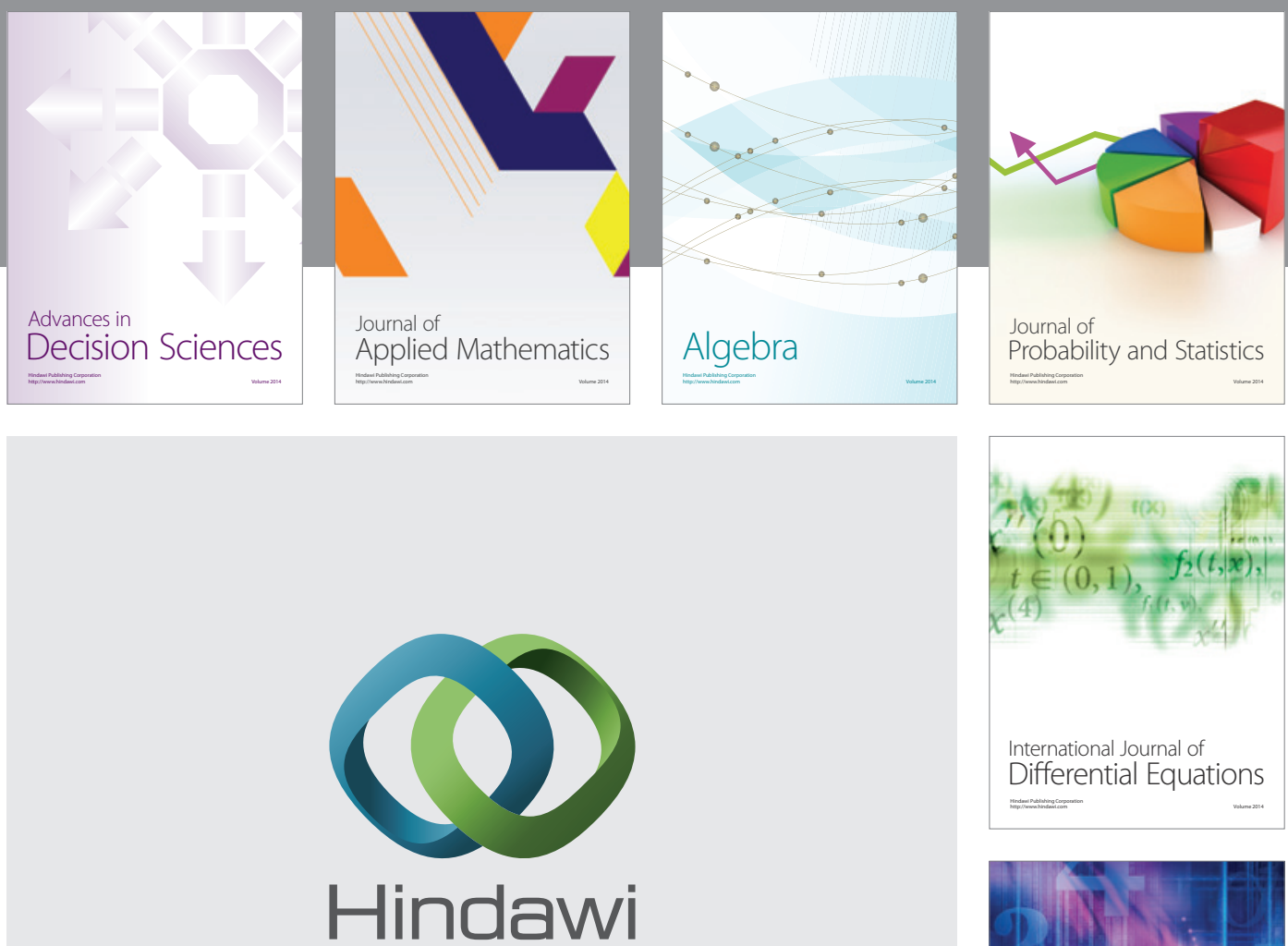

Submit your manuscripts at http://www.hindawi.com
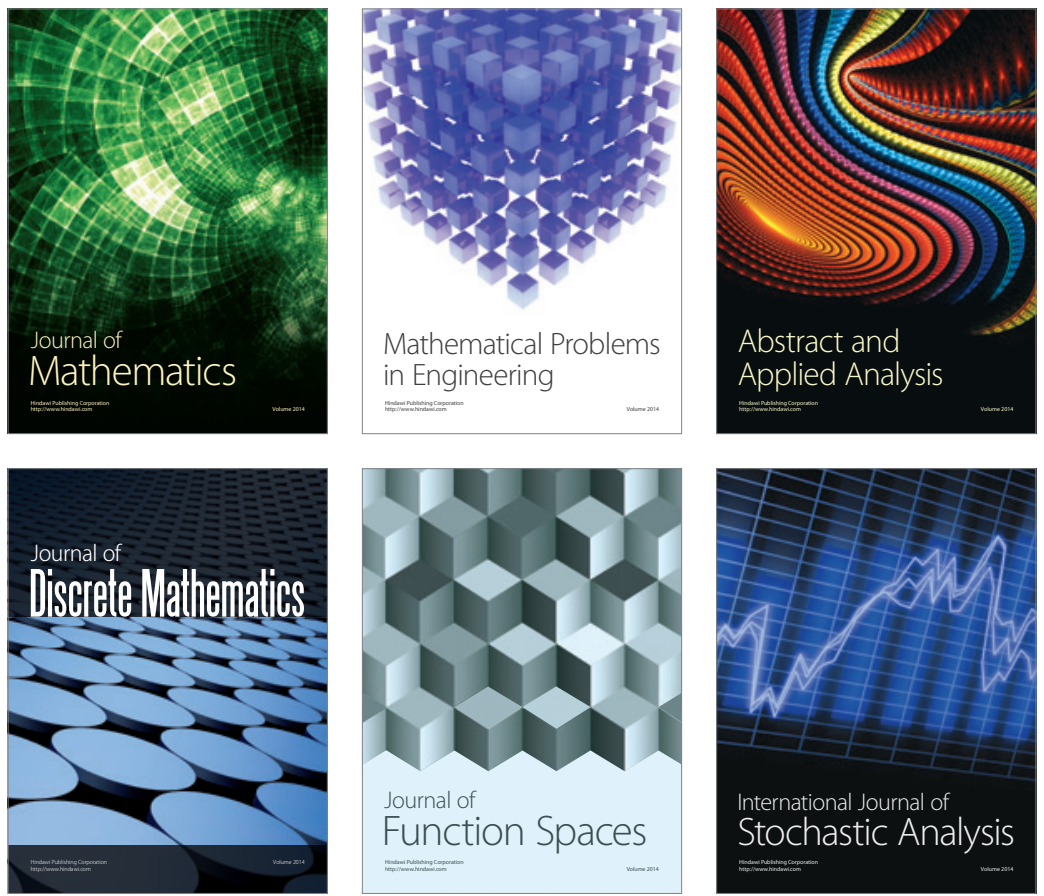

Journal of

Function Spaces

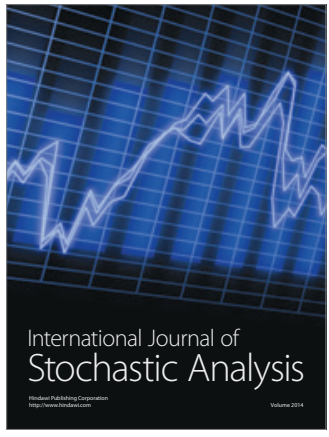

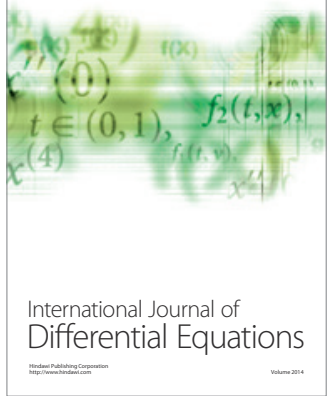
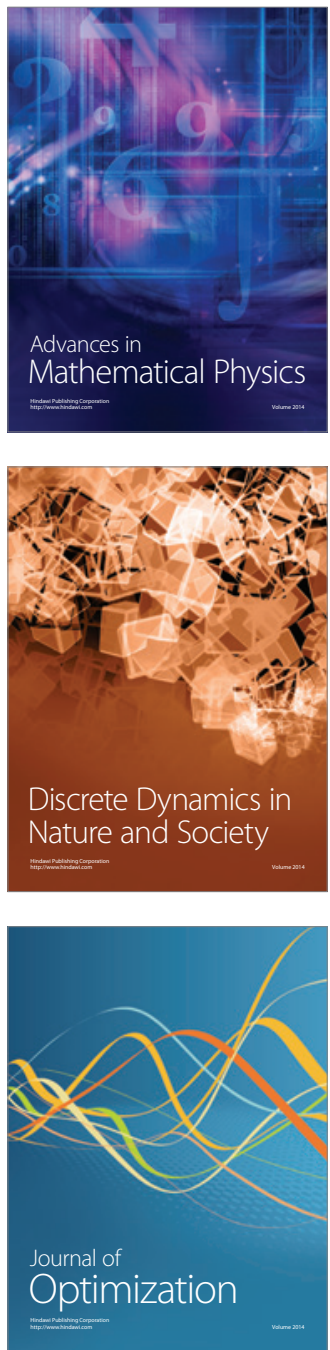